\title{
Total Knee Arthroplasty Complicated by a Severe Heterotopic Ossification: A Case Report
}

\author{
Antonio Spinarelli, ${ }^{1,}$ Massimiliano Carrozzo, ${ }^{1}$ Massimiliano Teti, ${ }^{1}$ Vittorio Nappi, ${ }^{1}$ and Biagio Moretti ${ }^{1}$ \\ ${ }^{1}$ Department of Neuroscience and Organs of Sense, Orthopaedic and Traumatology Unit, University of Bari, General Hospital, Bari, Italy \\ "Corresponding author: Antonio Spinarelli, Department of Neuroscience and Organs of Sense, Orthopaedic and Traumatology Unit, University of Bari, General Hospital, Bari, \\ Italy. Tel: +39-0805592719, Fax: +39-0805592713, E-mail: antoniospinarelli@gmail.com
}

Received 2016 January 15; Revised 2016 March 13; Accepted 2016 April 09.

\begin{abstract}
Introduction: Total knee arthroplasty (TKA) associated with heterotopic ossifications (HOs) are rare occurrences despite the relatively frequent surgery procedure of the hip arthroplasty. Up to present, different types of treatment were described in the literature. Case Presentation: We report an unusual case of TKA in a 70-year-old woman, which was early complicated by development of HO. It was successfully treated with a second surgery and a selective cyclooxygenase (COX)-2 inhibitor (celecoxib) with good clinical results.

Conclusions: This case illustrates that early operative and medical treatment can be used to optimally resolve stiffness caused by HO after total knee replacement.
\end{abstract}

Keywords: Heterotopic Ossification, Cox-2, Early Surgical Removal

\section{Introduction}

Stiffness following total knee arthroplasty (TKA) is disabling with pain that leads to a reduction of the function. So, many factors can contributed: patient factors, technical errors, local variables and postoperative complications (1). Severe heterotopic ossification (HO) is not a frequent reported event.

We report a 70-year-old woman with extensive $\mathrm{HO}$ and bony ankylosis of the knee after primary TKA.

\section{Case Presentation}

A 70-year-old woman had undergone primary TKA through traditional para-patellar approach (2) for the osteoarthritis of the right knee in the $29^{\text {th }}$ of January 2015 (Figure 1A - B). No complications were registered during hospital stay. The surgery procedure was performed by the first (A.S.) and the last (B.M.) authors under pneumatic tourniquet released before the quadriceps layer closure according to Barwell et al. (3).

The cemented implant used was Persona® Knee System (Zimmer®, Warsaw, Indiana, USA). A manual detachment of the extensor apparatus from the bone surface according to Tarabichi (4) was performed. The patella was not replaced but only reshaped as necessary with the resection of osteophytes. The wound healed normally and suture points were removed after 15 days from the surgery.
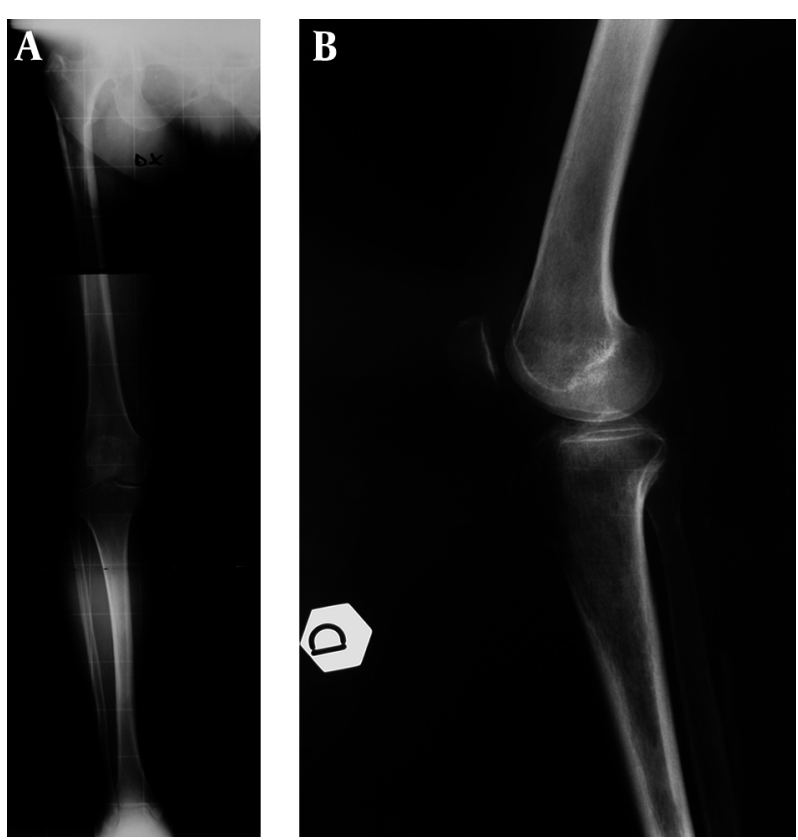

Figure 1. A and B, Preoperative radiographs of the right knee showing the osteoarthritis

The range of movement (ROM) of the right knee was $30^{\circ}$ to $75^{\circ}$ preoperatively and $0^{\circ}$ to $110^{\circ}$ immediately after TKA. The patient received 4000 UI of low-molecular- 
weight heparin for deep vein thrombosis prophylaxis 12 hours postsurgery for 35 days and our protocol for pain management.

Mobilization with a continuous passive motion device, with the flexion gradually increased, was begun after 4 - 5 hours postoperatively; after about 2 days, she was encouraged to move actively the operated joint, within tolerable pain limits, under the care of a physiotherapist. Partial weight bearing was begun at $2^{\text {th }}$ day postoperatively and the patient was discharged at day five after the surgery.

At 4 weeks after operation, she obtained a range of motion between $0^{\circ}$ to $90^{\circ}$; at 4 months the patient complained spontaneous knee pain with a restricted range of motion $\left(0^{\circ}-45^{\circ}\right)$. No trauma was reported to the knee. Blood test results were negative for erythrocyte sedimentation, C-reactive protein and white blood cells count. Also, the synovial fluid culture was negative.

At the examination, the knee appeared stable to the varus and valgus tests, with a flat scar, swollen and painful at the mobilization; the extension was complete. A mass was felt on direct painful palpation up to the distal part of quadriceps and near the proximal tip of the patella. Pain can be elicited also by knee extension against resistance.

The x-ray, in lateral and AP view, showed an HO graded III according to Rader (5), leaving from anterior femoral cortex along the extensor apparatus (Figure 2).

Dalury et al. (6) reported a radiographic grading system of cumulative heterotopic ossification: Type I patients had less than $2 \mathrm{~cm}$ total of new bone formation; type II had more than $2 \mathrm{~cm}$ of new bone formed, and type III had more than $5 \mathrm{~cm}$ of new bone formation.

After a review of the literature, a decision was made to treat the loss of range of motion with a surgical treatment and the use of a selective cyclooxygenase (COX)-2 inhibitor (celecoxib). So, the patient underwent a second surgery and, through longitudinal medial incision proximal to the patella, the HO was removed by osteotome and manually detached from surrounding muscles. At a macroscopic analysis, the $\mathrm{HO}$ appears $5.5 \mathrm{~cm}$. of diameter like a shell that surrounded the distal part of the femur that was classified as type III according to Dalury.

The intraoperative ROM was $0^{\circ}$ to $120^{\circ}$ (Figure $3 \mathrm{~A}$ - B). Copious irrigation with diluted betadine solution (17.5 cc of betadine for $500 \mathrm{cc}$ of $\mathrm{NaCl}$ solution) and an accurate suture on a suction drainage were performed. The postoperative radiographs are shown in Figure 4. Following the drainage tube removal after 24 hours from the surgery, the patient started ROM exercises with a continuous passive motion device supported by perineural antalgic catheter on femoral nerve.

The surgically removed tissue was histologically examined and was consistent with HO. The patient was dis- charged at day three after the surgery with a COX-2 inhibitor (100 mg day) and physiotherapy that consisted of daily session focusing on ROM exercises with additional daily session with continuous passive motion device. After 4 months from the second surgery, the pain control was optimal and there was a significant improvement in the $\operatorname{ROM}\left(0^{\circ}\right.$ to $\left.115^{\circ}\right)$ (Figure $\left.5 \mathrm{~A}-\mathrm{B}\right)$. No complications were registered.

\section{Discussion}

Heterotopic ossification is associated with numerous conditions, including hip surgery, spinal cord trauma, burns, head injury and fractures (7). It is the process by which mature lamellar bone is formed in soft tissues. It is the result of the differentiation of mesenchymal cells into osteoprogenitor cells, although the precise pathway is still unknown and debated $(8,9)$.

In the orthopedic surgery, heterotopic bone formation is a frequent complication described in shoulders, elbows, knees, hip sand spine. It is noted most frequently in the hips, in which the incidence of HO after total hip arthroplasty is $>50 \%$ (10), and then TKA has been reported to be between $4 \%$ and $42 \%(5,11,12)$.

As described by Chan et al. the etiology of knee stiffness after the TKA surgery is multifactorial with pre-, intra-, and post- operative factors (13). The authors affirm that the preoperative factors include limited ROM, prior surgery (14). Intra-operative factors include technical errors of the surgery with an improper balancing of the flexionextension gap, an oversizing or malpositioning of the implants and an inadequate femoral or tibial resection, creation of an anterior tibial slope, and inadequate resection of posterior osteophytes. The postoperative factors are the poor patient motivation to the physiotherapy, an early arthrofibrosis or subsequent infection, complex regional pain syndrome, and $\mathrm{HO}(13,14)$.

The presence of a pre-existing or contralateral knee $\mathrm{HO}$ is a risk factor for HO after TKA. Other risk factors include a hypertrophic or posttraumatic or postinfective knee osteoarthritis, previous knee surgery, and the concomitant conditions of diffuse idiopathic skeletal hyperostosis and ankylosing $(1,15)$.

Some technical errors during the surgery increase the risk of $\mathrm{HO}$ such as the splitting of the quadriceps tendon, stripping of anterior femoral periosteum, a too aggressive surgery without a sufficient soft tissues sparing and an inadequate hemostasis and irrigation useful to remove the excessive bone particles after osteotomy. Postoperatively the delayed mobilization, hematoma formation, and manipulation after the surgery also increase the risk of $\mathrm{HO}$ (13). 


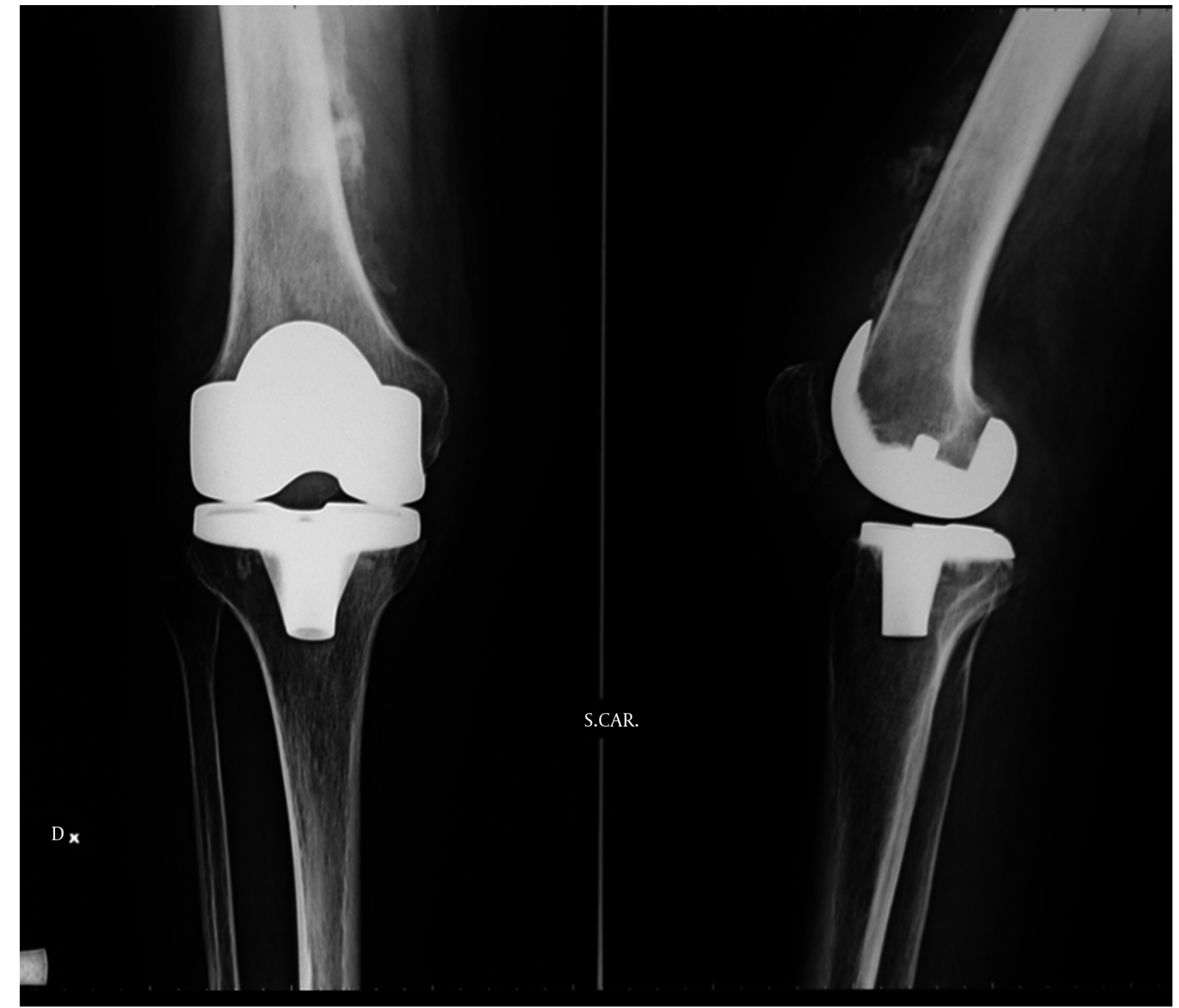

Figure 2. Presence of grade III heterotopic ossification anterior to the distal femur according to Rader et al. (5)

As described in other recent reports, the HO following primary TKA occurs with greater frequency maybe for the close scrutiny of the postoperative radiographs accounted for this relatively high prevalence (7). The localization of $\mathrm{HO}$ reported in our case is comparable to those reported in the literature $(6,7,11,13,16-19)$.

Several authors have stated that, since HO after TKA was founded to show no progression, and did not affect the final outcome neither prophylaxis nor specific therapy was required $(7,20)$. Hasegawa et al. (11) in their study showed in 2 of 10 knees the reabsorption of HO without surgical treatment, and the presence of small HO without knee flexion reduction in 9 knees. No statistical difference regarding the postoperative flexion between the knees with and without HO was found in their study.

Chan et al. (13) reported that a HO progressed for 9 years. They recommend surgical excision for very large symptomatic HO; however, they suggest manipulation under anesthesia followed aggressive physiotherapy is also recommended. Combination of surgery and radiotherapy is also suggested in their work $(13,21-24)$.

Our patient had a gradual worsening of the ROM and increase of the pain after 4 months from the surgery when she could not perform her normal daily activities. So many alternatives were described in the literature for the treatment of HOs. While postoperative radiotherapy has shown better results in the prophylaxis of the Hos (22), the use of shockwaves (25) and of NSAIDs (nonsteroidal anti- 

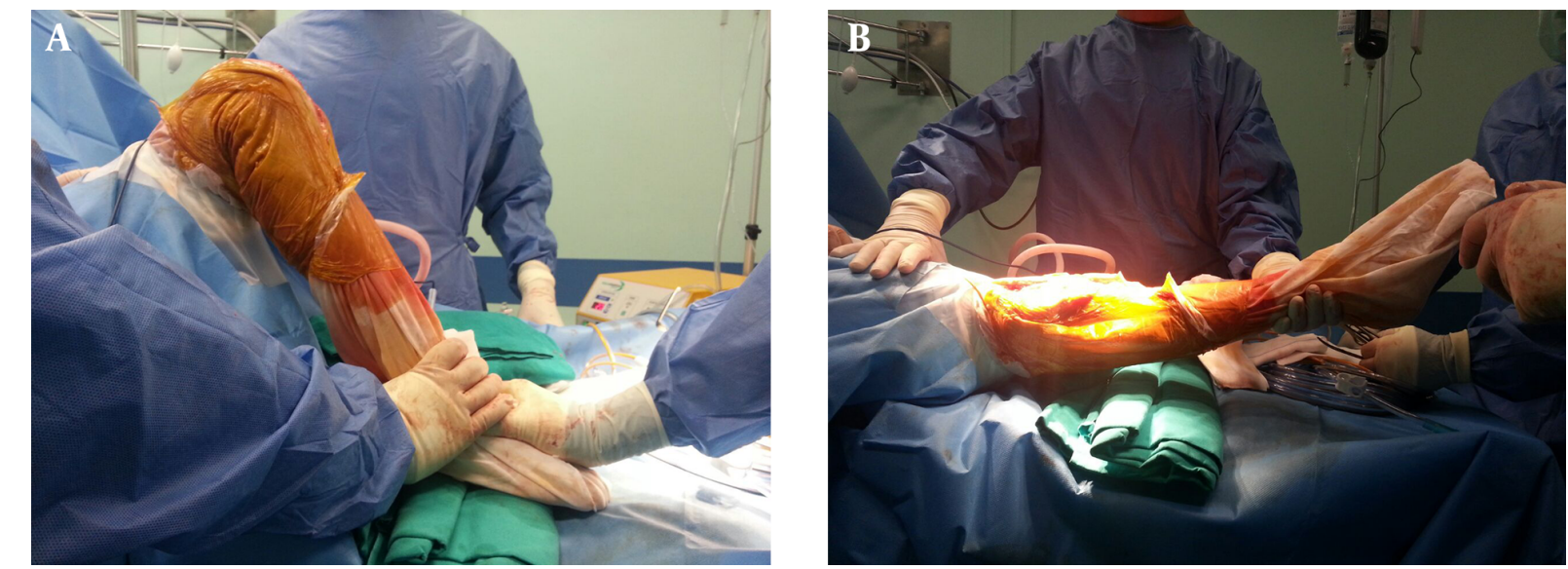

Figure 3. $A$ and $B$, Intraoperative flexion and extension

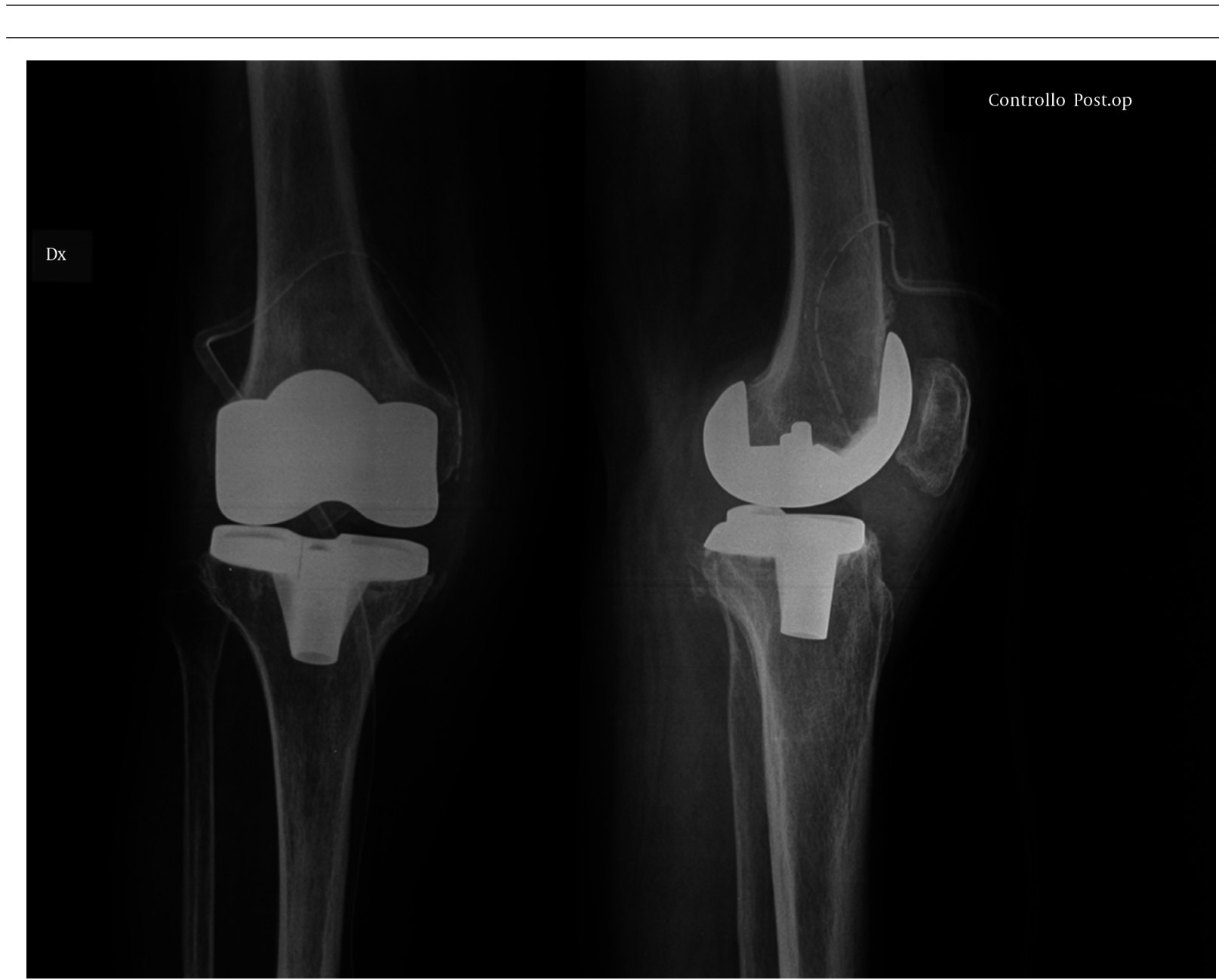

Figure 4. Postoperative radiographs of the heterotopic ossification removal 

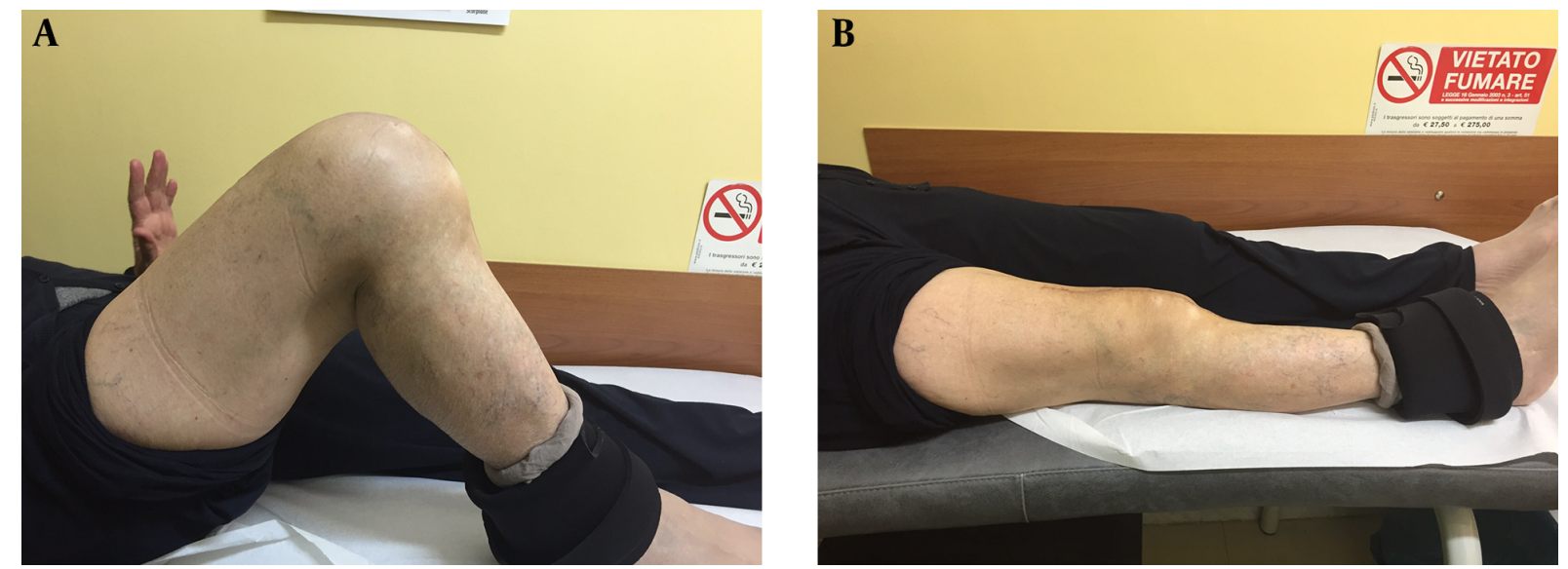

Figure 5. $A$ and $B$, Flexion and extension at four months

inflammatory drugs) registered better results in this issue (26). However, in more severe cases where the HOs are $>5$ $\mathrm{cm}$, and affect the ROM of the joint, this kind of treatment may be insufficient and surgical excision appears to be the only viable way to prevent stiffness of the prosthesis (24, 27). In the case report described, the surgical excision followed by a therapy with the celecoxib resulted in an early good outcome for the patient. We chose celecoxib because it shows the same efficacy as indomethacin in the prevention of HO after hip prosthesis with significantly fewer side effects (28).

Although the incidence of HO following total knee replacement is probably higher than what is commonly expected, the incidence of severe HO, which affects the ROM is much rarer with only a small number of reported cases. An optimal result was achieved via early surgical removal and celecoxib treatment that optimally resolved articular stiffness. We suggest this choice of treatment for HOs > $5 \mathrm{~cm}$ that limit the ROM and negatively influence the clinical outcomes of the TKR.

\section{Footnotes}

Conflict of Interests: The authors declare that there is no conflict of interests regarding the publication of this paper.

Financial Disclosure: The authors have no relevant financial or nonfinancial relationships to disclose.

\section{References}

1. Iorio R, Healy WL. Heterotopic ossification after hip and knee arthroplasty: risk factors, prevention, and treatment.J Am Acad Orthop Surg. 2002;10(6):409-16. [PubMed: 12470043].
2. Khan RJK, Keogh A, Fick DP, Wood DJ, Khan RJK. Surgical approaches in total knee arthroplasty. 2005 doi: 10.1002/14651858.cd005329.

3. Barwell J, Anderson G, Hassan A, Rawlings I. The effects of early tourniquet release during total knee arthroplasty: a prospective randomized double-blind study. J Bone Joint Surg Br. 1997;79(2):265-8. [PubMed: 9119854].

4. Tarabichi S, Tarabichi Y. Can an anterior quadriceps release improve range of motion in the stiff arthritic knee?. J Arthroplasty. 2010;25(4):571-5. doi: 10.1016/j.arth.2009.04.015. [PubMed: 19553069].

5. Rader CP, Barthel T, Haase M, Scheidler M, Eulert J. Heterotopic ossification after total knee arthroplasty. 54/615 cases after 1-6 years' followup. Acta Orthop Scand. 1997;68(1):46-50. [PubMed: 9057567].

6. Dalury DF, Jiranek WA. The incidence of heterotopic ossification after total knee arthroplasty. Arthroplasty. 2004;19(4):447-52. doi: 10.1016/j.arth.2003.12.064.

7. Furia JP, Pellegrini VD. Heterotopic ossification following primary total knee arthroplasty. Arthroplasty. 1995;10(4):413-9. doi: 10.1016/s0883-5403(05)80139-1.

8. Spinarelli A, Patella V, Petrera M, Abate A, Pesce V, Patella S. Heterotopic ossification after total hip arthroplasty: our experience. Musculoskelet Surg. 2011;95(1):1-5. doi: 10.1007/s12306-010-0091-6. [PubMed: 21210261].

9. Zychowicz ME. Pathophysiology of heterotopic ossification. Orthop Nurs. 2013;32(3):173-7. doi: 10.1097/NOR.0b013e3182920d85. [PubMed: 23695764] quiz 178-9.

10. Chidel MA, Suh JH, Matejczyk MB. Radiation prophylaxis for heterotopic ossification of the knee. J Arthroplasty. 2001;16(1):1-6. doi: 10.1054/arth.2001.16492. [PubMed: 11172262].

11. Hasegawa M, Ohashi T, Uchida A. Heterotopic ossification around distal femur after total knee arthroplasty. Arch Orthop Trauma Surg. 2002;122(5):274-8. doi: 10.1007/s00402-001-0377-0. [PubMed: 12070646].

12. Harwin SF, Stein AJ, Stern RE, Kulick RG. Heterotopic ossification following primary total knee arthroplasty. Arthroplasty. 1993;8(2):113-6. doi: 10.1016/s0883-5403(06)80048-3.

13. Chan PK, Chiu KY, Ng FY, Yan CH. Bony ankylosis of the knee secondary to heterotopic ossification after total knee arthroplasty: a case report. J Orthop Surg (Hong Kong). 2014;22(3):434-6. [PubMed: 25550034].

14. Bong MR, Di Cesare PE. Stiffness after total knee arthroplasty.J Am Acad Orthop Surg. 2004;12(3):164-71. [PubMed: 15161169].

15. Barrack RL, Brumfield CS, Rorabeck CH, Cleland D, Myers L. Heterotopic ossification after revision total knee arthroplasty. Clin Orthop Relat Res. 2002(404):208-13. [PubMed: 12439262]. 
16. Dodds AL, Keene GC. Severe Heterotopic Ossification following Total Knee Replacement. Case Rep Orthop. 2014;2014:265489. doi: 10.1155/2014/265489. [PubMed: 25431717].

17. Freedman EL, Freedman DM. Heterotopic ossification following total knee arthroplasty requiring surgical excision. Am J Orthop (Belle Mead NJ). 1996;25(8):559-61. [PubMed: 8871754].

18. Toyoda T, Matsumoto H, Tsuji T, Kinouchi J, Fujikawa K. Heterotopic ossification after total knee arthroplasty. J Arthroplasty. 2003;18(6):7604. [PubMed: 14513450].

19. Tigani D, Marinelli A, Bianchi G, Trentani P, Trentani F, Antonioli D. Heterotopic ossifications subsequent to knee arthroplasty. Chir Organi Mov. 2004;89(2):135-41. [PubMed: 15645790].

20. Daluga D, Lombardi AJ, Mallory TH, Vaughn BK. Knee manipulation following total knee arthroplasty. Analysis of prognostic variables. $J$ Arthroplasty. 1991;6(2):119-28. [PubMed: 1875202].

21. Kloth JK, Tanner M, Stiller W, Burkholder I, Kauczor HU, Ewerbeck V, et al. Radiation Dose Reduction in Digital Plain Radiography of the Knee after Total Knee Arthroplasty. Rofo. 2015;187(8):685-90. doi: 10.1055/s0034-1399559. [PubMed: 26090731].

22. Milakovic M, Popovic M, Raman S, Tsao M, Lam H, Chow E. Radiotherapy for the prophylaxis of heterotopic ossification: A systematic review and meta-analysis of randomized controlled trials. Radiother Oncol. 2015;116(1):4-9. doi:10.1016/j.radonc.2015.05.022.[PubMed: 26163090].

23. Devnani AS. Management of heterotopic ossification affecting both hips and knees. Singapore Med J. 2008;49(6):501-4. [PubMed: 18581026].

24. Chen S, Yu SY, Yan H, Cai JY, Ouyang Y, Ruan HJ, et al. The time point in surgical excision of heterotopic ossification of post-traumatic stiff elbow: recommendation for early excision followed by early exercise. J Shoulder Elbow Surg. 2015;24(8):1165-71. doi: 10.1016/j.jse.2015.05.044 [PubMed: 26189802].

25. Choi YM, Hong SH, Lee CH, Kang JH, Oh JS. Extracorporeal shock wave therapy for painful chronic neurogenic heterotopic ossification after traumatic brain injury: a case report. Ann Rehabil Med. 2015;39(2):31822. doi: 10.5535/arm.2015.39.2.318. [PubMed: 25932431].

26. Kan SL, Yang B, Ning GZ, Chen LX, Li YL, Gao SJ, et al. Nonsteroidal Anti-inflammatory Drugs as Prophylaxis for Heterotopic Ossification after Total Hip Arthroplasty: A Systematic Review and Meta-Analysis. Medicine (Baltimore). 2015;94(18):e828. doi: 10.1097/MD.0000000000000828. [PubMed: 25950691].

27. Edwards DS, Barbur SA, Bull AM, Stranks GJ. Posterior mini-incision total hip arthroplasty controls the extent of post-operative formation of heterotopic ossification. Eur J Orthop Surg Traumatol. 2015;25(6):1051-5. doi: 10.1007/s00590-015-1646-x. [PubMed: 25953634].

28. Romano CL, Duci D, Romano D, Mazza M, Meani E. Celecoxib versus indomethacin in the prevention of heterotopic ossification after total hip arthroplasty. J Arthroplasty. 2004;19(1):14-8. [PubMed:14716644]. 